\title{
ON THE INVERSION INVARIANCE OF INVARIANT MEANS
}

\author{
R. G. DOUGLAS
}

An invariant mean on a group $G$ is a normalized, positive, translation invariant linear functional defined on the space of all bounded complex valued functions on $G$. Some groups possess an invariant mean (or are said to be amenable), while others do not. In particular, all abelian groups are amenable [2, \$17.5].

An invariant mean on a group need not be inversion invariant. It is quite easy to show, however, that if a group is amenable, then there exists an invariant mean that is also inversion invariant $[2, \$ 17.19 \mathrm{a}]$. The purpose of this note is to prove that on a nontorsion abelian group, there must exist invariant means that are not inversion invariant. More specifically it is shown that no "elementary" (or extremal) invariant mean on a nontorsion abelian group is inversion invariant.

For a finite abelian group, there exists a unique invariant mean which must be inversion invariant. For an infinite torsion abelian group, it is not clear just what the situation is. (See Remark 4.)

While this note concerns itself only with the question of the inversion invariance of invariant means defined on the space of all bounded functions on an abelian group, this question is perhaps of even more interest in the more general context of invariant means defined on some "suitable" subspace of functions on an arbitrary (perhaps topological) group. The known results seem to indicate that this question may be related to the question of uniqueness of invariant means.

Let $G$ be an abelian group and let $B(G)$ denote the complex Banach space of bounded complex valued functions on $G$ in which $\|f\|$ $=\sup \{|f(x)| \mid x \in G\}$. An element $L \in B(G) *$ (the conjugate space of $B(G)$ ) is said to be an invariant mean on $G$ if (1) $\|L\|=1$; (2) $L f \geqq 0$ for $f \geqq 0$; and (3) $L f_{x}=L f$ for each $x \in G$ and $f \in B(G)$, where $f_{x}(y)=f(x y)$ for $y \in G .^{1}$ An abelian group always has an invariant mean; for this result and further information about invariant means consult $[2$, $\$ 17]$.

For each function $f \in B(G)$, let $f^{*}$ denote the function defined on $G$ such that $f^{*}(x)=f\left(x^{-1}\right)$ for $x \in G$. Similarly, for each functional $L \in B(G)^{*}$, let $L^{*}$ denote the functional defined on $B(G)$ such that $L^{*}(f)=L\left(f^{*}\right)$ for each $f \in B(G)$. A functional $L \in B(G)^{*}$ is said to be inversion invariant if $L=L^{*}$. If $L$ is an invariant mean on $G$, then the

Received by the editors May 23, 1964.

${ }^{1}$ Multiplicative notation will be used for the group operation throughout this note. 
functional $M=\frac{1}{2}\left\{L+L^{*}\right\}$ is also an invariant mean that is in addition inversion invariant.

A couple of lemmas used in proving the main theorem will be stated after the following notation is introduced. Let $\chi_{E}$ denote the characteristic function of the subset $E$ of $G$, that is, $\chi_{E}(x)$ is defined to be 1 if $x \in G$ and 0 otherwise.

LEMMA 1. Let $G$ be an abelian group and $L$ be an invariant mean on $G$. Suppose $\Sigma$ is a sub-semigroup of $G$ that generates $G$. Then the linear functional $M$ defined on $B(G)$ such that $M f=L\left(\chi_{\Sigma} f\right)$ for $f \in B(G)$ is translation invariant and positive.

Thus either $M$ is the zero functional or $\|M\|^{-1} M$ is an invariant mean on $G$.

Proof. If $x \in \Sigma$ and $n$ is a non-negative integer, then $x^{n+1} \Sigma \subset x^{n} \Sigma$ and if $Q_{n}=x^{n} \Sigma-x^{n+1} \Sigma$ (set theoretic difference), then the sets $\left\{Q_{n}\right\}_{n=0}^{\infty}$ are pairwise disjoint. Moreover, because $x Q_{n}=Q_{n+1}$, it follows that $L\left(\chi_{Q_{n}}\right)=L\left(\chi_{Q_{0}}\right)$ for all $n$, and hence

$$
1=L\left(\chi_{G}\right) \geqq L\left(\chi_{\Sigma}\right) \geqq \sum_{n=1}^{N} L\left(\chi_{Q_{n}}\right)=N L\left(\chi_{Q_{0}}\right) \geqq 0 .
$$

Thus $L\left(\chi_{(\Sigma-x \Sigma)}\right)=L\left(\chi_{Q_{0}}\right)=0$.

The linear functional $M$ is translation invariant. To see this, suppose $x \in \Sigma$ and $f \in B(G)$; then

$$
M\left(f_{x}\right)=L\left(\chi_{\Sigma} f_{x}\right)=L\left(\chi_{x} \varepsilon f\right)=L\left(\chi_{\Sigma} f\right)+L\left(\chi_{(\Sigma-x \Sigma)} f\right)=L\left(\chi_{\Sigma} f\right)=M f
$$

If $z \in G$ and $f \in B(G)$, then there exist $x$ and $y$ in $\Sigma$ such that $z=x y^{-1}$ and $M\left(f_{z}\right)=M\left(\left(f_{z}\right)_{y}\right)=M\left(f_{x}\right)=M(f)$. Thus $M$ is translation invariant. That $M$ is positive is obvious.

REMARK 1. This proof is almost identical to that used in $[1, \mathrm{pp}$. 518-519] to yield a different result.

REMARK 2. This lemma as well as the next is valid for non-abelian groups.

LEMMA 2. Let $G$ be an abelian group, $H$ a subgroup of $G$ and $L$ an invariant mean on $G$. Then $L\left(\chi_{H}\right)=1 /[G: H]$, where $[G: H]$ denotes the number of cosets of $G / H$.

Proof. Obvious.

In order to make possible the statement of a stronger theorem, the following terminology is needed. A linear functional $L \in B(G)^{*}$ is said to be supported on the subset $E$ of $G$, if $L(f)=L\left(\chi_{E} f\right)$ for every $f \in B(G)$. The linear functionals $L_{1}$ and $L_{2}$ in $B(G)^{*}$ are said to be strongly singular if there exist disjoint subsets $E$ and $F$ of $G$ such that 
$L_{1}$ is supported on $E$ and $L_{2}$ is supported on $F$.

THEOREM. Let $G$ be a nontorsion abelian group and $L$ be an invariant mean on $G$ that is also inversion invariant. Then $L=\frac{1}{2}\left(M+M^{*}\right)$, where $M$ is an invariant mean for which $M$ and $M^{*}$ are strongly singular.

Proof. Let $H$ denote the subgroup of $G$ that consists of the elements of finite order. Then $G / H \neq\{H\}$ is a torsion-free abelian group. Thus there exists a sub-semigroup $\Sigma_{0}$ of $G / H$ such that $\Sigma_{0} \cap \Sigma_{0}^{-1}=\{H\}$ and $\Sigma_{0} \cup \Sigma_{0}^{-1}=G / H[3,8.1 .2]$. Let $\Sigma$ denote the inverse image of $\Sigma_{0}$ in $G$ under the canonical map. Then $\Sigma$ is a subsemigroup of $G$, that generates $G$, and $\Sigma \cap \Sigma^{-1}=H$. Therefore, it follows from Lemma 1 that $M_{0}(f)=L\left(\chi_{\Sigma} f\right)$ is a translation invariant positive functional on $B(G)$. Moreover, from Lemma 2 it follows that $L\left(\chi_{H}\right)=0$ and thus that $L\left(\chi_{(\Sigma-H)}\right)=L\left(\chi_{\left(\Sigma^{-1}-H\right)}\right)$. Hence $M_{0}\left(\chi_{G}\right)=\frac{1}{2}$ and $M=2 M_{0}$ is an invariant mean. Moreover, $L=\frac{1}{2}\left(M+M^{*}\right)$ and $M$ and $M^{*}$ are supported on the disjoint subsets $\Sigma-H$ and $\Sigma^{-1}-H$.

An invariant mean on $G$ is said to be elementary if it is an extreme point of the $w^{*}$-compact convex subset of invariant means.

COROLlaRY. If $G$ is a nontorsion abelian group and $L$ is an elementary invariant mean on $G$, then $L$ is not inversion invariant.

COROLlaRy. Let $G$ be a nontorsion abelian group. Then there exists an invariant mean on $G$ that is not inversion invariant.

REMARK 3. The argument used to prove the theorem can also be used to prove a similar result for a large subclass of the amenable nonabelian groups. The interested reader can formulate the precise statement of these results.

The author is indebted to the referee for the following example.

REMARK 4. The hypothesis in the Theorem and its corollaries that $G$ be a nontorsion abelian group cannot be omitted. There exist infinite torsion abelian groups for which all invariant means are inversion invariant. An easy example of such a group is one in which every element is of order two. In this case, $f=f^{*}$ for all $f$ and thus each invariant mean is inversion invariant. The product of infinitely many copies of the cyclic group of order two is an infinite abelian group in which every element has order two.

\section{REFERENCES}

1. M. M. Day, Amenable semigroups, Illinois J. Math. 1 (1957), 509-544.

2. E. Hewitt and K. A. Ross, Abstract harmonic analysis. I, Grundlehren der Mathematischen Wissenschaften, Bd. 115, Springer, Berlin, 1963.

3. W. Rudin, Fourier analysis on groups, Interscience, New York, 1962.

UNIVERSITY OF MichigAN 